\title{
Demodulation System of the FBG Reflectance Spectrum Based on Optical Low Coherence Reflectometry
}

\author{
L.Y. Zhang, X.Y. Shen, J. Sun, J.C. Hu, Y. Han \\ College of Metrology \& Measurement Engineering \\ China Jiliang University \\ China
}

\begin{abstract}
The key to the complex sensor detection(such as the non homogenous strain detection) of the fiber Bragg grating(FBG) is the demodulation of FBG reflectance spectrum. This paper designs the demodulation system which is based on the optical low coherence reflectometry $(\mathrm{OLCR})$ princple. The system is mainly composed of the improved OLCR set-up and the LabVIEW demodulation program. The experimental results show that the demodulation system can effectively reduce the noise and amplify the interference signal, quickly demodulate the FBG reflectance spectrum and exactly find the Bragg wavelength after the zero padding and Gauss curve fitting, especially the repeatability can reach to $4 \mathrm{pm}$.
\end{abstract}

Keywords-FBG; OLCR; demodulation; Bragg wavelength; repeatability

\section{INTRODUCTION}

Due to the advantages of light quality, small size, high sensitivity, anti electromagnetic interference and so on[1], FBGs have been widely used in the sensor detection. Because of some complex changed physics are measured through the varied FBG reflectance spectrum, the key to the sensors is the demodulation of reflectance spectrum. The traditional basic way to achieve the reflectance spectrum is using the optical spectrum analyzer(OSA), but the higher resolution and accuracy OSA is quite expensive and cannot directly get the varied physics. However, nowadays the reflectometric techniques which include OLCR [2,3], optical frequency domain reflectometry(OFDR) $[4,5]$ and optical time domain reflectometry(OTDR) [6] can successfully be used in FBGs detecting. And among them, the OLCR is the most popular and effective one. Now the researches on OLCR mainly focus on the function application, but actually there is much scope to improve the properties of OLCR system used in FBG detecting. For examples, the repeatability of demodulated uniform FBG wavelength can only reach to $23 \mathrm{pm}$ according to [7], the resolution of FBG reflectance spectrum can only reach to $3 \mathrm{pm}$ according to [8]. Hence, we design the demodulation system of FBG reflectance spectrum with optimized OLCR set-up and corresponding LabVIEW program.

\section{THEORY OF OLCR}

The simplified OLCR setup is essentially a scanning Michelson interferometer coupled with a broadband and low- coherence light source such as super luminescent diode(SLD). Figure 1 presents the basic all-fiber Michelson type OLCR setup for FBG characterization. The light launched by SLD propagate through the $3 \mathrm{~dB} 2 \times 2$ optical coupler, and split into the so-called reference and test arm later. The two reflected light from the movable mirror and FBG recombined again in the coupler, and finally directed to the detector. The optical path difference between two arms varied by changing the position of the mirror. Only when the path difference is shorter than the coherence length [9] of the light Source, the interference signal will occur.

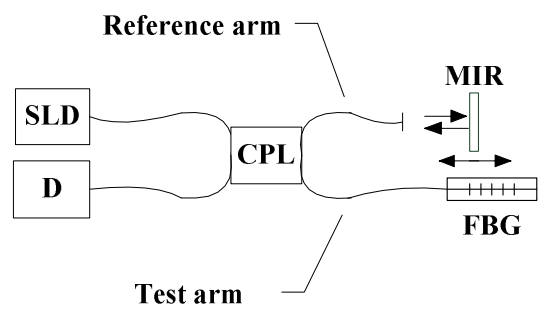

FIGURE I. BASIC OLCR SET-UP FOR FBG CHARACTERIZATION

When we do not take into account the light attenuation along the OLCR set-up, the detected intensity of the interference signal is

$$
I(\delta)=\frac{1}{2}\left|E_{r}(\delta)+E_{t}\right|^{2}=\frac{1}{2}\left\{\left|E_{r}(\delta)\right|^{2}+\left|E_{t}\right|^{2}\right\}+\operatorname{Re}\left(E_{r}(\delta) E_{t}^{*}\right)
$$

where $E_{\mathrm{r}}$ and $E_{\mathrm{t}}$ are the reflected electrical fields of reference and test arms, $\delta$ is the optical path diference between two arms. But the intensity measured by the detector include AC interference part(we do not need) and DC interference part, and also the interference signal can easily be influenced by the noise. Hence, we improve the OLCR set-up by optimizing the structure and programming for the control and demodulation so that it become a effectively and exactly demodulation system of FBG. 


\section{DEMODULATION SYSTEM}

\section{A. Improved OLCR Set-up}

The new OLCR set-up of our demodulation system is composed of the SLD, balanced detecting structure(BD Structure), reference arm with airbone translation stage(ATS), collimating lens(LENS) and mirror(MIR), test arm with polarization controller(POLA) and FBG, data acquision card(DAC), and finally the PC(Figure 2).

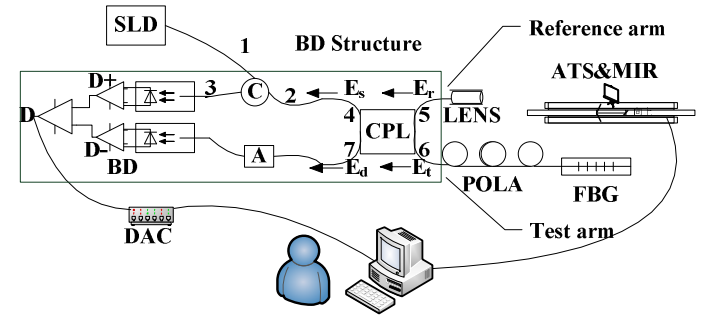

FIGURE II. FBG DEMODULATION SYSTEM BASED ON THE IMPROVED OLCR SET-UP

The LENS can guarantee the maximum spatial coupling efficiency of the light, and the ATS can reduce the effect of vibration. The POLA placed in test arm modifies the polarization state angle $\theta$ between two arms, and the measured AC intensity is effected by the factor $\cos (\theta)$ [10]. The balanced detector can obtain the maximum $\mathrm{AC}$ interference signal when the POLA is manually set to achieve $\theta=0$. The rectangle part is the $\mathrm{BD}$ structure which includes the circulator(C), coupler(CPL), attenuator(A), a balanced detector(BD) composed of two similar diodes(D+ and D-) and the differential processing module(D). The attenuator used after the port 7 of coupler works for compensating the insertion loss of the circulator. Owing to the properties of the 2 $\times 2$ coupler [11] and the differential detection structure, the balanced detection can realize. The intensities correspond to the port 4 and 7 are

$$
\begin{aligned}
& I_{s}(\delta)=\frac{1}{2}\left(I_{r}+I_{t}\right)+\operatorname{Re}\left(\int d \sigma E_{r}(\delta) \bullet E_{t}^{*} \bullet e^{i \pi / 2}\right) \\
& I_{d}(\delta)=\frac{1}{2}\left(I_{r}+I_{t}\right)+\operatorname{Re}\left(\int d \sigma E_{r}(\delta) \bullet E_{t}^{*} \bullet e^{-i \pi / 2}\right)
\end{aligned}
$$

where $\sigma$ is the wavemunber, and obviously the DC part is identical but that the $\mathrm{AC}$ part has a $\pi$ phase factor difference. Then the differential intensity is obtained

$$
I_{\text {diff }}=I_{s}-I_{d}=I_{O L C R}(\delta)=2 \operatorname{Re}\left(\mathrm{e}^{-i \pi / 2} \int d \sigma E_{r}(\delta) \bullet E_{t}^{*}\right)
$$

Benefit from the new OLCR set-up, we can achieve the double interference intensity signal. Meantime the noise is suppressed, and the signal to noise ratio(SNR) is improved $[12,13]$, the calculation formulation is

$$
S N R_{\text {Busic }}=\frac{\alpha^{2} P_{R} P_{T}}{\left\{\frac{2 k_{B} T}{R_{L}}+e \alpha P_{R}+\frac{\left(1+V^{2}\right)}{3 \Delta V} \alpha^{2}\left(P_{R}+P_{T}\right)^{2}\right\} B}
$$

$$
S N R_{\text {improcoed }}=\frac{\alpha^{2} P_{R} P_{T}}{\left\{\frac{2 k_{B} T}{R_{L}}+e \alpha P_{R}+\frac{2\left(1+V^{2}\right)}{3 \Delta V} \alpha^{2} P_{R} P_{T}\right\} B}
$$

Where $\alpha=\eta \mathrm{e} / \mathrm{h} v_{0}, \eta$ is the photoelectric conversion efficiency, $\mathrm{e}$ is the electron charge and $\mathrm{h}$ is the Planck constant, $\Delta v$ and $v_{0}$ are FWHM and center frequency of source light, $P_{\mathrm{R}}$ and $P_{\mathrm{T}}$ are power of reference and test arm, $K_{\mathrm{B}}$ is Boltzmann constant, $T$ is Kelvin temperature, $R_{\mathrm{L}}$ is load resistance, $V$ is source degree of polarization and $B$ is the bandwidth of detector.

\section{B. Program of System}

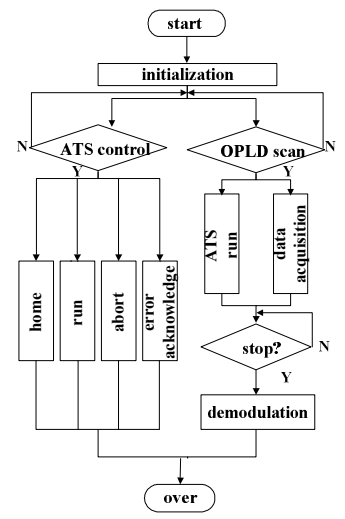

FIGURE III. MAIN PROGRAM FLOW

All the control and demodulation program is designed by LabVIEW, figure 3 is the main program flow. After the initialization, the program can be choosed to carry out either independent airbone stage move or optical path length scan. The stage move mainly includes the options of home to zero, run, abort and error acknowledge.

During the scanning process, the producer and consumer structure of program based on the queue can realize the synchronous operation of stage moving and datas acquiring. As soon as the datas acquisition stops, the demodulation of FBG reflectance spectrum starts. The specific demodulation process can describe as figure 4.

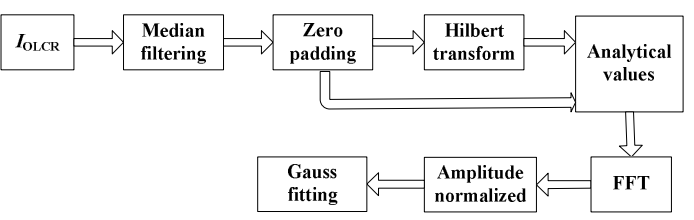

FIGURE IV. MAIN DEMODULATION PROCESS

According to [7], the interference signal $I_{\mathrm{OLCR}}$ is proportional to the FBG reflectance spectrum. After median filtering of the time domain signal, program try to improve the spectral resoulation through padding zeros after the interference signal[8]. The datas after zero padding and the Hilbert transform one are combined to create the analytical values. Finally the gauss fit of the normalized amplitude can result to the better repeatability. 


\section{EXPERIMENTS AND DISCUSSIONS}

\section{A. Interference Signal}

The scheme of BD structure shows that the theoretically measured interference signal should be twice of the basic OLCR set-up, but due to numerous external factors such as the fiber disturbance, properties of diodes, environment factors and so on, the actual obtained signal is amplified but not equal to the double one.

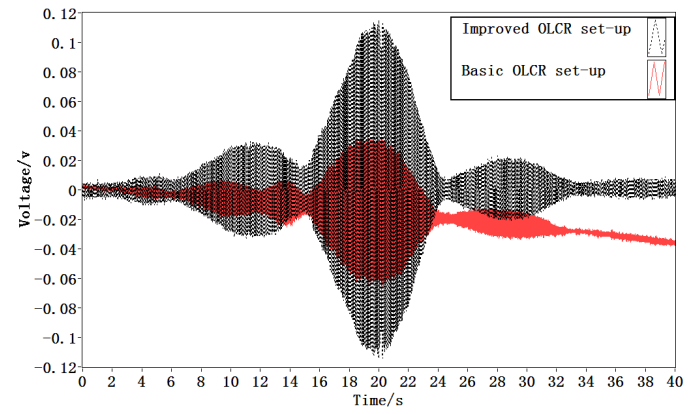

FIGURE V. INTERFERENCE SIGNAL OF OLCR SET-UP

Figure 5 is the interference signal of the basic OLCR setup and the improved one. Obviously the interference signal of improved OLCR set-up is amplified and symmetrical to the Y axis. The basic OLCR interference signal is shifted closely to zero of $\mathrm{Y}$ axis. The signal is asymmetric due to the decaying interference intensity result from the existent angle between mirror and output light. When the angle is small, the influence can be reduced by the new set-up. The fiber disturbance and human factors lead to the datas distortion left of the basic OLCR signal.

\section{B. $\quad$ Suppressed Noise and SNR}

The SNR is strongly related to the detector noise which depends on the reference power, the detection scheme of chosen improved OLCR set-up improves the SNR. When $\eta=0.8, \quad \Delta v=64.5 \mathrm{~nm}, \quad v_{0}=1563.4 \mathrm{~nm}, \quad P_{\mathrm{R}}=200 \sim 300 \mathrm{uW}, \quad P_{\mathrm{T}}$ $=6.76 \mathrm{uW}, T=300 \mathrm{~K}, R_{\mathrm{L}}=50 \Omega, V=1, B=200 \mathrm{MHz}$, the $\mathrm{SNR}$ can be obtained in figure 6 .

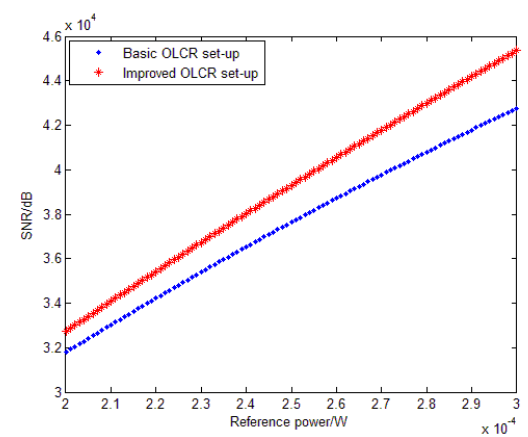

FIGURE VI. SIGNAL TO NOISE RATIO

It is easily to find that the SNR advantage of the improved OLCR set-up increases as long as the reference power raises. The noise is effectively suppressed.

C. Reflectance Spectrum and Repeatability of Bragg Wavelength

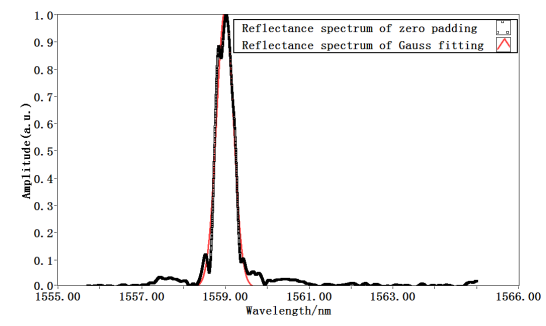

FIGURE VII

$$
\text { E SPECTRUM }
$$

REFLECTANC

According to the demodulation process described as figure 4 , we improve the spectral resoulation to $1 \mathrm{pm}$ by padding zeros, and fit the Gauss reflectance spectrum based on the discrete points. Both the reflectance spectrum are shown in the figure 7. There is a subprime peak and vally beside the maximum value due to that the FBG is not absolute uniform.

In the table 1 are 10 Bragg wavelengths of the Gauss-fitting demodulated from nurmous datas. Refer to the national metrology standards $[14,15]$, the repeatability can be calculated by the Bessel formula, and the results show that the repeatability can reach to $4 \mathrm{pm}$.

TABLE I. THE BRAGG WAVELENGTH OF THE GAUSS-FITtING

\begin{tabular}{|c|c|c|c|}
\hline No. & $\begin{array}{c}\text { Bragg } \\
\text { wavelength/nm }\end{array}$ & Residual/nm & Repeatability/nm \\
\hline 1 & 1558.994 & 0.006 & \multirow{10}{*}{0.004} \\
\hline 2 & 1558.983 & -0.005 & \\
\hline 3 & 1558.991 & 0.003 & \\
\hline 4 & 1558.984 & -0.004 & \\
\hline 5 & 1558.992 & 0.004 & \\
\hline 6 & 1558.985 & -0.003 & \\
\hline 7 & 1558.987 & -0.001 & \\
\hline 8 & 1558.987 & -0.001 & \\
\hline 9 & 1558.989 & 0.001 & \\
\hline 10 & 1558.983 & -0.005 & \\
\hline
\end{tabular}

Note: The average wavelength is $1558.988 \mathrm{~nm}$.

\section{CONCLUSION}

We have shown the demodulation system of FBG reflectance spectrum based on the OLCR which is composed of the improved OLCR set-up and the LabVIEW program. And the given experimental results proved that the demodulation system can amplify the interference signal, at the same time the noise are suppressed. Also the reflectance spectrum can easily be obtained, and the repeatability of Bragg wavelength can reach to $4 \mathrm{pm}$. This automatic and accurate demodulation system can be used for complex sensor detecting such as non homogeneous strain detecting later on.

\section{ACKNOWLEDGEMENTS}

Project supported by the National Natural Science Foundation of China (Grant No.61205082 ); Project supported by the National Natural Science Foundation of Zhejiang Province of China (Grant No.LQ14F050004 ) 


\section{REFERENCES}

[1] Li, H.N., \& Ren, L., Structural Health Monitoring-Fiber Grating Sensing Technology, China-Building: Beijing, pp. 4-5, 2008.

[2] Coric, D., Limberger, H.G., \& Salathe, R.P., Distributed measurements of fiber birefringence and diametric load using optical low-coherence reflectometry and fiber gratings. Optics Express., 14(24), pp. 1180511813, 2006

[3] Lu, P., \& Chen, Q.Y., Optical low-coherence reflectometry for deflection measurement with a fiber Bragg grating cantilever sensor, Meas. Sci. Technol., 20(2009), pp. 1-8, 2009.

[4] MacDonald, R.I., Frequency domain optical reflectometer. Appl. Opt., 20(10), pp. 1840-1844, 1981.

[5] Soller, B., Gifford, D., \& Wolfe, M., High resolution optical frequency domain reflectometry for characterization of components and assemblies Opt. Exp., 13(2), pp. 666-674, 2005

[6] Aoyama, Y., Nakagawa, K., \& Itoh, T., Optical time domain reflectometry in a single-mode fiber. IEEE J. Quantum Electron, 17(6), pp. 862-868, 1981.

[7] Obaton, A.F., Palavicini, C., \& Jaouen, Y., Characterization of fiber bragg grating by phase-sensitive optical low-coherence reflectometry. IEEE Transactions on Instrumentation and Measurement., 55(5), pp. 1696-1703, 2006

[8] Li, G.S., Li, Q., \& Chen, Z.M., Measurement of FBG reflection spectra by white light interferometry. Laser Technology, 37(1), pp. 20-23, 2013.

[9] Saleh, B.E.A., \& Teich, M.C., Fundamental of photonics. John Wiley\&Sons, pp. 348-352, 1991.

[10] Dong, H.C., Liu, Y., \& Y, K., Theory analysis of polarized beam coherent combination. Chinese Journal of Lasers, 36(9), pp. 2346-2351, 2009.

[11] Zhang, J.H., Effect of loss on output phase differences of optical fiber couplers. Optical Fiber \& Electric Cable, 6(6), pp. 17-21, 1999.

[12] Zhu, X.N., Mao, Y.X., \& Liang, Y.M., Noise analyses of optical coherence tomography system( I )-theories and calculations. ACTA PHOTONICA SINICA, 36(3), pp. 452-456, 2007.

[13] Zhu, X.N., Mao, Y.X., \& Liang, Y.M., Noise analyses of optical coherence tomography system( II )-Fourier domain and time domain OCT systems. ACTA PHOTONICA SINICA, 36(3), pp. 457-461, 2007.

[14] JJF 1033-2008, Rule for the examination of measurement standard[S].

[15] JJF 1001-2011, General terms in metrology and their definitions[S]. 